\title{
Electron-Tracking Compton Gamma-Ray Camera for Small Animal and Phantom Imaging
}

\author{
Shigeto Kabuki, ${ }^{\mathrm{a},}$ Hiroyuki Kimura, ${ }^{\mathrm{b}}$ Hiroo Amano, ${ }^{\mathrm{b}}$ Yuji Nakamoto, ${ }^{\mathrm{c}}$ Hidetoshi \\ Kubo, ${ }^{a}$ Kentaro Miuchi, ${ }^{a}$ Shunsuke Kurosawa, ${ }^{a}$ Michiaki Takahashi, ${ }^{a}$ Hidekazu \\ Kawashima, ${ }^{\mathrm{c}}$ Masashi Ueda, ${ }^{\mathrm{d}}$ Tomohisa Okada, ${ }^{\mathrm{c}}$ Atsushi Kubo, ${ }^{\mathrm{e}}$ Etuso Kunieda, ${ }^{\mathrm{e}}$ \\ Tadaki Nakahara, ${ }^{e}$ Ryota Kohara, ${ }^{f}$ Osamu Miyazaki, ${ }^{\mathrm{f}}$ Tetsuo Nakazawa, ${ }^{\mathrm{f}}$ Takashi \\ Shirahata, ${ }^{\mathrm{f}}$ Etsuji Yamamoto, ${ }^{\mathrm{f}}$ Koichi Ogawa, ${ }^{\mathrm{g}}$ Kaori Togashi, ${ }^{\mathrm{c}}$ Hideo Saji, ${ }^{\mathrm{b}}$ and Toru \\ Tanimori, ${ }^{\text {a }}$ \\ ${ }^{a}$ Department of Physics, Gradulate School of Science, Kyoto University, Kyoto 606-8502, Japan \\ ${ }^{b}$ Department of Patho-functional Bioanalysis, Graduate School of Pharmaceutical Sciences, Kyoto University, Kyoto 606-8501, Japan \\ ${ }^{c}$ Department of Diagnostic Imaging and Nuclear Medicine, Kyoto University, Kyoto 606-8507, Japan \\ ${ }^{d}$ Radioisotopes Research Labaoratory, Kyoto University Hospital, Kyoto 606-8507, Japan \\ ${ }^{e}$ Department of Radiology, Keio University School of Medicine, Tokyo 160-8582, Japan \\ ${ }^{f}$ Application Development Office, Hitachi Medical Corporation, Chiba 277-0804, Japan \\ ${ }^{g}$ Department of Electronic Informatics Faculty of Engineering Hosei University Tokyo 184-8584, Japan
}

Elsevier use only: Received date here; revised date here; accepted date here

\begin{abstract}
We have developed an electron-tracking Compton camera (ETCC) for medical use. Our ETCC has a wide energy dynamic range (200-1300 keV) and wide field of view (3 steradian), and thus has potential for advanced medical use. To evaluate the ETCC, we imaged the head (brain) and bladder of mice that had been administered with F-18-FDG. We also imaged the head and thyroid gland of mice using double tracers of F-18-FDG and I-131 ion.
\end{abstract}

Keywords: Compton camera; Molecular imaging; TPC; LaBr3

* Corresponding author. Tel.: +81-75-753-3728; fax:+81-75-753-3799 ; e-mail:kabuki@cr.scphys.kyoto-u.ac.jp . 


\section{Introduction}

In nuclear medicine, a molecule that is labeled with a radioisotope (RI) is administered to a human, and gamma rays from the RI are imaged with a gamma camera. Newer molecular imaging techniques are now under study, and recently much effort has gone into developing detectors and chemical probes for such imaging. The applicability of molecular probes to conventional imaging such as positron emission tomography (PET) and single photon emission computed tomography (SPECT) has also been studied. PET detects gamma rays by annihilation of a positron and electron pair, but the energy dynamic range for PET is only $511 \mathrm{keV}$. Because a collimator is required, the energy dynamic range for SPECT is less than $365 \mathrm{keV}$. These energy limitations are a major problem in the design of new imaging reagents.

The Compton camera has a wide energy dynamic range and wide field of view [1, 2, 3]. We have developed an advanced Compton camera, called the electron-tracking Compton camera (ETCC), which can detect the recoil electron track. The ETCC can visualize an image even with low statistics using this electron track information [4, 5, 6, 7].

\section{Detector and Analysis}

The ETCC consists of two detectors. An initial gamma ray is scattered in the first detector, which is a micro time projection chamber ( $\mu$-TPC) based on the proportional gaseous pixel chamber micro gas chamber $(\mu$-PIC) $[8,9]$. The $\mu$-TPC (size $10 \times 10 \times$ $10 \mathrm{~cm}^{3}$ ) can catch the 3D recoil electron track [10]. The scattered gamma ray is caught by the second detector, which is composed of pixel scintillator arrays (PSAs). We use four $\mathrm{LaBr}_{3}$ :Ce PSAs; pixel size of three arrays are $6 \times 6 \times 15 \mathrm{~mm}^{3}$ [11] and 1 array is $4 \times 4 \times 30 \mathrm{~mm}^{3}$ [12]. Whole PSA area is 10 $\times 10 \mathrm{~cm}^{2}$ (Fig. 1A). Our ETCC system size including all electronics is $100 \times 60 \times 180 \mathrm{~cm}^{3}$ (Fig. 1B).

The ETCC can reconstruct the gamma-ray direction, event by event, using information from the electron track and the absorption point of the scattered gamma ray.

Conventional Compton cameras cannot detect the electron track. Without this information, the gammaray direction is reconstructed as a ring shape. In contrast, our camera can determine the arc shape using electron track information.

We use the list-mode maximum-likelihood expectation-maximization (list-mode MLEM) method as a reconstruction filter [13]. This method is commonly used for medical imaging. The ETCC's spatial resolution at $511 \mathrm{keV}$ is $11 \mathrm{~mm}$ (FWHM) at $10 \mathrm{~cm}$ from the detector surface. Its acceptance uniformity at $365 \mathrm{keV}$ over FOV $\left(20 \times 20 \mathrm{~cm}^{2}\right)$ is $11 \%(1 \sigma)$ at $10 \mathrm{~cm}$ from the detector surface.

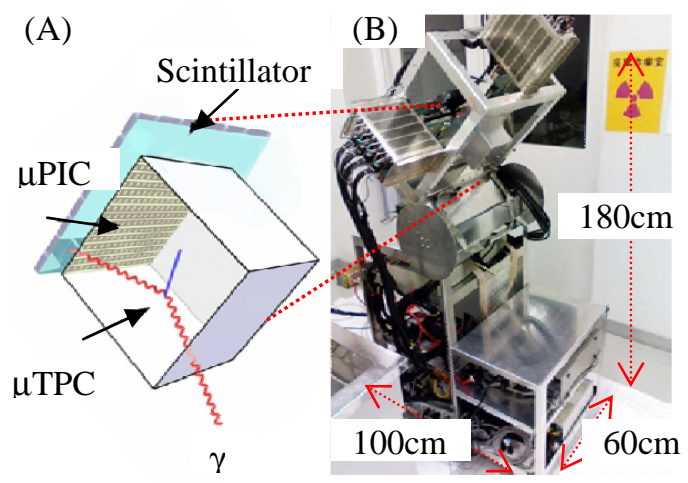

Fig 1. (A): Schematic view of the ETCC. The straight line is the electron track; the wavy line is the initial and scattered gamma ray. (B): Photograph of the ETCC. System size including all electronics is $180 \times 60 \times 100 \mathrm{~cm}^{3}$.

\section{Imaging Results}

We took images of small animals to evaluate our ETCC camera. ETCC imaging was performed on tumor-bearing mice. We selected the HeLa and put tumors on both shoulders. Two hours after intervenous injection of F-18-Fludeoxyglucose (F18-FDG, $49 \mathrm{MBq}$ ), the mice were sacrificed with Nembutal ("Nembutal" is a registered trademark of Abbot Laboratories Corp.), and ETCC imaging was performed for 16 hours. ETCC data were fused with MRI (T2-weighted imaging) anatomical data to determine the location of radionuclide uptake. PET imaging was performed to compare with the ETCC image. The ETCC image (Fig. 2A) clearly shows the brain, tumor, heart, and bladder uptake, in a manner 

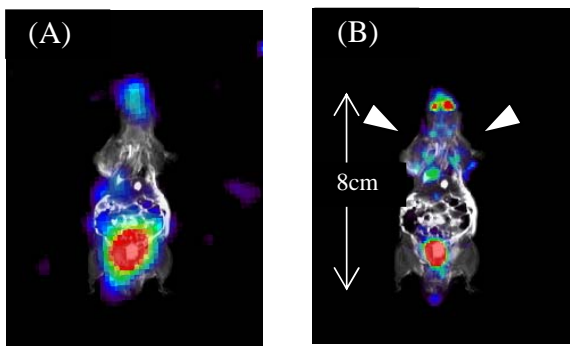

Fig. 2. (A): F-18-FDG fusion image of ETCC (color contour) and MRI (monochrome contour). (B): F-18-FDG fusion image of PET (color contour) and MRI (monochrome contour). The white arrows show the tumor position.

similar to the PET image (Fig. 2B). These data indicates that ETCC works correctly.

We next performed multiple-nuclide imaging. We administered F-18-FDG (10 MBq) and I-131 ion (3 $\mathrm{MBq})$ at the same time to mice that had the HeLa tumor on both shoulders. Three hours after vein injection, the mice were sacrificed with Nembutal, and ETCC imaging was performed for 19 hours. ETCC data show that I-131 ion accumulated in the thyroid gland and bladder (Fig. 3A), and F-18-FDG accumulated in the brain, tumor, heart, and bladder (Fig. 3B). The fused image of ETCC and MRI (Fig. 3C) shows that ETCC succeeded in double-tracer imaging and that the reported distributions of F-18FDG and I-131 ion are correct. Thus, using the ETCC, we succeeded in visualizing clearly the targeted areas.

\section{Conclusion}

We developed the ETCC for medical use and succeeded in imaging the bodies of mice. F-18-FDG was administered to HeLa-tumor-bearing mice, and the ETCC was used to image the head and bladder. When these data are fused with anatomical data from MRI, the distribution of F-18-FDG is observed to be correct and in agreement with the PET data.

In addition, we also succeeded in double-tracer imaging using the F-18-FDG and I-131 ion, and can clearly recognize their differences in each image. Our

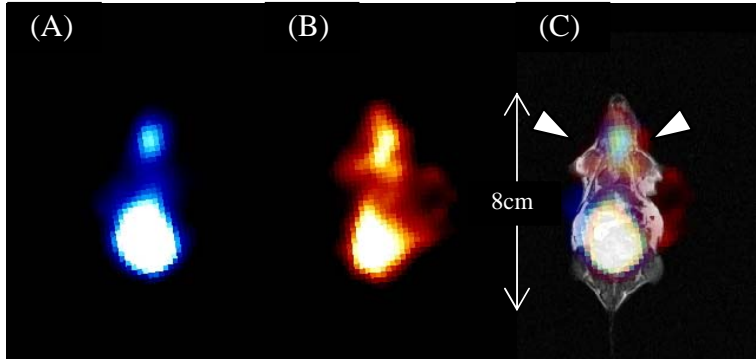

Fig. 3. (A): I-131 ion image of ETCC. (B): F-18-FDG image of ETCC. (C): Fusion image of ETCC (color contour) and MRI (monochrome contour). The white arrows show the tumor position.

ETCC is thus useful for imaging small animals over a wide energy dynamic range. These results are also promising for the design of new imaging reagents.

\section{Acknowledgments}

This work was supported by a Grant-in-Aid for Scientific Research from the Japan Ministry of Education, Culture, Science, Sports and Technology (SENTAN) of the Japan Science and Technology Agency, and a Grant-in-Aid for the Global COE "Next Generation Physics, Spun from Universality and Emergence.”

\section{References}

[1] R. Todd, J. Nightingale, and D. Everett, Nature, 251 (1974) 132

[2] M. Singh and D. Doria, Medical Physics 10 (1983) 4

[3] S. Motomura et al., Pure Appl. Chem, 8012 (2008) 2657

[4] S. Kabuki et al., Nucl. Instr. and Meth. A, 580 (2007) 1031

[5] T. Tanimori et al., New Astro. Rev, 48 (2004) 236

[6] A. Takada et al., Nucl. Instr. Meth. A, 546 (2005) 820

[7] R. Orito et al., Nucl. Instr. Meth. A, 513 (2003) 408

[8] T. Nagayoshi et al. Nucl. Instr. Meth. A, 546 (2005) 457

[9] A. Ochi et al., Nucl. Instr. Meth. A, 471 (2001) 264

[10] H. Kubo et al., Nucl. Instr. Meth. A, 513 (2003) 94

[11] H. Kubo et al., IEEE NSS Conference record, (2007) 4569

[12] A. Kuhn et al., IEEE Trans. Nucl. Sci., 51 (2004) 2550

S. J. Wilderman et al., IEEE Trans. Nucl. Sci., 45 (1998) 957 